\title{
Permanence and Extinction of Stochastic Logistic System with Feedback Control under Regime Switching
}

\author{
Hongxiao $\mathrm{Hu}^{1}$ and Ling $\mathrm{Zhu}^{2}$ \\ ${ }^{1}$ College of Science, University of Shanghai for Science and Technology, Shanghai 200093, China \\ ${ }^{2}$ School of Science, Anhui Agriculture University, Heifei 230036, China \\ Correspondence should be addressed to Hongxiao Hu; hhxiao1@126.com
}

Received 27 July 2015; Revised 2 September 2015; Accepted 10 September 2015

Academic Editor: Seenith Sivasundaram

Copyright @ $2015 \mathrm{H}$. Hu and L. Zhu. This is an open access article distributed under the Creative Commons Attribution License, which permits unrestricted use, distribution, and reproduction in any medium, provided the original work is properly cited.

\begin{abstract}
We study a stochastic logistic system with feedback control under regime switching. Sufficient conditions for extinction, nonpersistence in the mean, weak persistence, and persistence in the mean are established. A very important fact is found in our results; that is, the feedback control is harmless to the permanence of species even under the regime switching and stochastic perturbation environments. Finally, some examples are introduced to illustrate the main results.
\end{abstract}

\section{Introduction}

The classical logistic equation with feedback control is

$$
\begin{aligned}
& \dot{x}(t)=x(t)(r(t)-a(t) x(t)-c(t) u(t)), \\
& \dot{u}(t)=-e(t) u(t)+f(t) x(t),
\end{aligned}
$$

where $x(t)$ denotes the population size at time $t$ and $u(t)$ is an "indirect control" variable (see [1]). It has been studied extensively and many important results on the global dynamics of solutions have been founded (see [2-5] and references therein). On the other hand, population systems in the real world are often affected by environmental noise and there are various types of environmental noise, for example, white or color noise (see [6-14] and references therein) and it has been shown that the presence of such noise affects population systems significantly. But the white and color noise are unobservable, and we can only observe the species $x(t)$. Hence, we can use the same feedback control with system (1) to regulate the species which is affected by the environmental noises. To the best of the authors' knowledge, few scholars still consider the stochastic perturbation logistic system with feedback controls under regime switching. And we have known very little about how the feedback control affects the survival of species which is under the random factors and switching environment.
In this paper, motivated by the above analysis, we will study the following stochastic logistic system with feedback control under regime switching:

$$
\begin{aligned}
& \mathrm{d} x(t) \\
& =x(t)(r(\gamma(t))-a(\gamma(t)) x(t)-c(\gamma(t)) u(t)) \mathrm{d} t \\
& \quad+\sigma(\gamma(t)) x(t) \mathrm{d} B(t), \\
& \dot{u}(t)=-e(t) u(t)+f(t) x(t),
\end{aligned}
$$

where $\gamma(t)$ is a Markov chain on the state space $\mathbb{S}$ as defined in the next section, for each $i \in \mathbb{S}, r(i)$ represents the intrinsic growth rate, $r(i) / a(i)$ is the carrying capacity in regime $i, a(i)$, $c(i)$, and $\sigma(i)$ are nonnegative constants, and $e(t)$ and $f(t)$ are nonnegative continuous bounded functions on $[0,+\infty)$.

In this work, our purpose is to design feedback controls such that the system becomes permanent or extinct. We will establish the sufficient conditions for extinction, nonpersistence in the mean, weak persistence, and persistence in the mean of system (2).

\section{Main Results}

Throughout this paper, unless otherwise specified, let $(\Omega, \mathscr{F}$, $\left.\left\{\mathscr{F}_{t}\right\}_{t>0}, \mathbb{P}\right)$ be a complete probability space with filtration 
$\left\{\mathscr{F}_{t}\right\}_{t \geq 0}$ satisfying the usual conditions (i.e., it is rightcontinuous and $\mathscr{F}_{0}$ contains all $P$-null set). Let $B(t), t \geq 0$, be 1-dimension standard Brownian motion defined on this probability space. We also denote by $R_{+}$the interval $[0,+\infty)$ and denote by $R_{+}^{2}$ the set $\{(x, y) \mid x>0, y>0\}$. Let $\gamma(t)$ be a right-continuous Markov chain on the probability space, taking values in a finite-state space $\mathbb{S}=\{1,2, \ldots, n\}$ for all $t \geq 0$, with the generator $\Gamma=\left(\gamma_{i j}\right)_{n \times n}$ given by

$$
\begin{aligned}
\mathbb{P}\{\gamma(t+\Delta t)=j \mid \gamma(t)=i\} & \begin{cases}\gamma_{i j} \Delta t+o(\Delta t), & \text { if } j \neq i, \\
1+\gamma_{i i} \Delta t+o(\Delta t), & \text { if } j=i,\end{cases}
\end{aligned}
$$

where $\gamma_{i j} \geq 0$ for $i, j=1,2, \ldots, n$ with $j \neq i$ and $\sum_{j=1}^{n} \gamma_{i j}=0$ for $i=1,2, \ldots, n$. We assume that the Markov chain $\gamma(\cdot)$ is independent of the Brownian motion $B(\cdot)$. As a standing hypothesis, we assume in this paper that the Markov chain $\gamma(t)$ is irreducible; that is, for any $i, j \in \mathbb{S}$, one can find finite numbers $i_{1}, i_{2}, \ldots, i_{k} \in \mathbb{S}$ such that $\gamma_{i, i_{1}} \gamma_{i_{1}, i_{2}} \gamma_{i_{k}, j}>0$. Under this condition, the Markov chain has a unique stationary distribution $\pi=\left(\pi_{1}, \pi_{2}, \ldots, \pi_{n}\right)$ which can be obtained by solving the following linear equation $\pi \Gamma=0$ subject to $\sum_{i=1}^{n} \pi_{i}=1$ and $\pi_{i}>0$ for all $i \in \mathbb{S}$. For convenience and simplicity in the following discussion, define

$$
\begin{aligned}
& v_{u}=\max _{i \in \mathbb{S}} v(i), \\
& v_{l}=\min _{i \in \mathbb{S}} v(i),
\end{aligned}
$$

and we also define

$$
\begin{aligned}
f_{u} & =\sup _{s \in R_{+}} f(s), \\
f_{l} & =\inf _{s \in R_{+}} f(s), \\
\langle f\rangle^{*} & =\limsup _{t \rightarrow \infty} \frac{1}{t} \int_{0}^{t} f(s) \mathrm{d} s, \\
\langle f\rangle_{*} & =\liminf _{t \rightarrow \infty} \frac{1}{t} \int_{0}^{t} f(s) \mathrm{d} s,
\end{aligned}
$$

where $f(s)$ is a bounded function on $R_{+}$.

In system (2), $x(t)$ is the size of the species and $u(t)$ is the regulator; thus, we are only interested in the positive solutions. Moreover, in order for a stochastic differential equation to have a unique global (i.e., no explosion in a finite time) solution for any given initial value, the coefficients of the equation are generally required to satisfy the linear growth condition and local Lipschitz condition (cf. Mao [15]). However, the coefficients of system (2) do not satisfy the linear growth condition, though they are locally Lipschitz continuous. In this section, using the comparison theorem of stochastic equations (see [16]), we will show there is a unique positive solution with positive initial value of system (2).

Theorem 1. For any given initial value $\left(x_{0}, u_{0}\right) \in R_{+}^{2}$, there is a unique continuous positive solution $(x(t), u(t))$ to system (2) on $t \geq 0$ a.s.

Proof. Since the coefficients of the equation are locally Lipschitz continuous, it is known that for any given initial value $\left(x_{0}, u_{0}\right) \in R_{+}^{2}$ there is a unique maximal local solution $(x(t), u(t))$ for all $t \in\left[0, \tau_{e}\right)$, where $\tau_{e}$ is the explosion time. Furthermore, by Theorem 2.1 in [17], we have

$$
\begin{aligned}
& x(t)=\frac{\exp \left\{\int_{0}^{t}[b(\gamma(s))-c(\gamma(s)) u(s)] \mathrm{d} s+\int_{0}^{t} \sigma(\gamma(s)) \mathrm{d} B(s)\right\}}{1 / x_{0}+\int_{0}^{t} a(\gamma(s)) \exp \left\{\int_{0}^{s}[b(\gamma(\tau))-c(\gamma(s)) u(s)] \mathrm{d} \tau+\int_{0}^{s} \sigma(\gamma(\tau)) \mathrm{d} B(\tau)\right\} \mathrm{d} s}>0, \\
& u(t) \geq u_{0} \exp \left\{-\int_{0}^{t} e(s) \mathrm{d} s\right\}>0,
\end{aligned}
$$

where $b(i)=r(i)-0.5 \sigma^{2}(i)$ for each $i \in \mathbb{S}$. Hence, to show this solution is global positive, we only show that $\tau_{e}=\infty$ a.s. By the first equation of (2), we have
Consider the following auxiliary equation:

$\forall t \in\left[0, \tau_{e}\right)$

$$
\begin{aligned}
\mathrm{d} x(t) \leq & x(t)(r(\gamma(t))-a(\gamma(t)) x(t)) \mathrm{d} t \\
& +\sigma(\gamma(t)) x(t) \mathrm{d} B(t) .
\end{aligned}
$$

$$
\begin{aligned}
\mathrm{d} y(t)= & y(t)(r(\gamma(t))-a(\gamma(t)) y(t)) \mathrm{d} t \\
& +\sigma(\gamma(t)) y(t) \mathrm{d} B(t), \\
y(0)= & x_{0} .
\end{aligned}
$$


From Theorem 2.1 in [17], we known that there exists a unique continuous positive solution $y(t)$ of system (8) for any positive initial value $x_{0}$, which will remain in $R_{+}$with probability one. Consequently, by the comparison theorem of stochastic differential equation, we have

$$
x(t) \leq y(t) \quad \forall t \geq 0 \text { a.s. }
$$

Therefore, $x(t)<\infty$ for all $t>0$ a.s. By the second equation of (2), we can represent $u(t)$ by

$$
\begin{aligned}
u(t)= & \left(\int_{0}^{t} f(s) x(s) \exp \left\{\int_{0}^{s} e(\tau) \mathrm{d} \tau\right\}+u_{0}\right) \\
& \cdot \exp \left\{-\int_{0}^{t} e(s) \mathrm{d} s\right\} .
\end{aligned}
$$

From this, we can find that if $x(t)$ is global, then $u(t)$ also is a global solution; that is, $\tau_{e}=\infty$ a.s. This completes the proof of the theorem.

Now, we will discuss extinction and persistence of system (2). For convenience and simplicity in the following discussion, we denote $b(i)=r(i)-0.5 \sigma^{2}(i)$ and $\widetilde{b}=\sum_{i=1}^{n} \pi_{i} b(i)$ for $i \in \mathbb{S}$ and write $(x(t), u(t))=\left(x\left(t, 0, x_{0}, u_{0}\right), u\left(t, 0, x_{0}, u_{0}\right)\right)$ simply for any $\left(x_{0}, u_{0}\right) \in R_{+}^{2}$. Applying Itô's formula to $\ln x(t)$, we have that

$$
\begin{aligned}
\mathrm{d} \ln x(t) & \\
= & (b(\gamma(t))-a(\gamma(t)) x(t)-c(\gamma(t)) u(t)) \mathrm{d} t \\
& +\sigma(\gamma(t)) \mathrm{d} B(t) .
\end{aligned}
$$

Then, we have

$$
\begin{aligned}
& \ln x(t) \\
& =\ln x_{0} \\
& \quad+\int_{0}^{t}(b(\gamma(s))-a(\gamma(s)) x(s)-c(\gamma(s)) u(s)) \mathrm{d} s \\
& \quad+M(t),
\end{aligned}
$$

where $M(t)=\int_{0}^{t} \sigma(\gamma(s)) \mathrm{d} B(s)$. By the second equation of system (2), we have

$$
u(t)-u_{0}=-\int_{0}^{t} e(s) u(s) \mathrm{d} s+\int_{0}^{t} f(s) x(s) \mathrm{d} s .
$$

Note that $M(t)$ is a local martingale. Making use of the strong law of large numbers for local martingales (see Mao [15]), we have

$$
\lim _{t \rightarrow \infty} \frac{M(t)}{t}=0 \quad \text { a.s. }
$$

We denote $\Omega_{0}=\left\{\lim _{t \rightarrow \infty} M(t) / t=0\right\}$; obviously, $\mathbb{P}\left(\Omega_{0}\right)=1$.

Theorem 2. If $\tilde{b}<0$ and there is a positive constant $\lambda$ such that

$$
\liminf _{t \rightarrow \infty} \int_{t}^{t+\lambda} e(s) \mathrm{d} s>0
$$

then system (2) will go to extinction almost surely; that is, $\lim _{t \rightarrow \infty} x(t)=0$ and $\lim _{t \rightarrow \infty} u(t)=0$ a.s.

Proof. For any $\omega \in \Omega_{0}$, from (12), we have

$$
\frac{\ln x(t, \omega)}{t} \leq \frac{\ln x_{0}}{t}+\frac{1}{t} \int_{0}^{t} b(\gamma(s)) \mathrm{d} s+\frac{M(t, \omega)}{t} .
$$

Making use of (14) and the ergodicity, we obtain $\limsup \sup _{t \rightarrow \infty} \ln x(t, \omega) / t \leq \tilde{b}<0$. That is, we say $\lim _{t \rightarrow \infty} x(t, \omega)=0$. Now, we will prove $\lim _{t \rightarrow \infty} u(t, \omega)=0$. Since $\lim _{t \rightarrow \infty} x(t, \omega)=0$, then, for any $\alpha_{0}>0$, there is a positive constant $T_{0}$ such that $|x(t, \omega)|<\alpha_{0} / f_{u}$ for all $t \geq T_{0}$. Consequently, from (13), we have that

$$
\frac{\mathrm{d} u(t, \omega)}{\mathrm{d} t} \leq-e(t) u(t, \omega)+\alpha_{0}, \quad \forall t \geq T_{0}
$$

We consider the comparison equation

$$
\frac{\mathrm{d} v(t)}{\mathrm{d} t}=-e(t) v(t)+\alpha_{0}
$$

By Lemma 2.1 of [5] and (15), we have that for any positive constant $\varepsilon$ there are constants $\delta=\delta(\varepsilon)$ and $T_{1}=T_{1}\left(u\left(T_{0}\right)\right)>$ $T_{0}$ such that when $\left|\alpha_{0}\right|<\delta$, we have $|v(t)|<\varepsilon$ for all $t \geq T_{1}$, where $v(t)$ is the solution of system (18) with initial condition $v\left(T_{0}\right)=u\left(T_{0}, \omega\right)$. Therefore, by the comparison theorem, we obtain $u(t, \omega)<\varepsilon$ for all $t \geq T_{1}$. Since $\varepsilon$ is arbitrary, we have $\lim _{t \rightarrow \infty} u(t, \omega)=0$. Since $\mathbb{P}\left(\Omega_{0}\right)=1$, this completes the proof of the theorem.

Theorem 3. If $a_{l}>0, e_{l}>0$ and $\tilde{b}=0$, then system (2) will be nompersistent in the mean a.s.; that is, $\lim _{t \rightarrow \infty} \int_{0}^{t} x(s) \mathrm{d} s / t=0$ and $\lim _{t \rightarrow \infty} \int_{0}^{t} u(s) \mathrm{d} s / t=0$ a.s.

Proof. $\widetilde{b}=0$ and (14) imply that, for any $\varepsilon>0$ and $\omega \in \Omega_{0}$, there is a positive constant $T_{0}$ such that

$$
\begin{aligned}
\frac{1}{t} \int_{0}^{t} b(\gamma(s)) \mathrm{d} s & \leq \lim _{t \rightarrow \infty} \frac{1}{t} \int_{0}^{t} b(\gamma(s)) \mathrm{d} s+\frac{\varepsilon}{2}=\widetilde{b}+\frac{\varepsilon}{2} \\
& =\frac{\varepsilon}{2} \\
M(t, \omega) & \leq \frac{\varepsilon t}{2}
\end{aligned}
$$

Then, it follows from (12) that $\ln x(t, \omega) \leq \ln x_{0}+\varepsilon t-$ $a_{l} \int_{0}^{t} x(s, \omega) \mathrm{d} s$. Let $h(t)=\int_{0}^{t} x(s, \omega) \mathrm{d} s$; then, we have deduced that $e^{a_{l} h(t)} \mathrm{d} h(t) \leq x_{0} e^{\varepsilon t} \mathrm{~d} t$. Integrating this inequality from $T>T_{0}$ to $t$ results in $e^{a_{l} h(t)} \leq e^{a_{l} h(T)}+x_{0} a_{l}\left(e^{\varepsilon t}-e^{\varepsilon T}\right) / \varepsilon$. It follows that

$$
h(t) \leq \frac{1}{a_{l}} \ln \left(e^{a_{l} h(T)}+\frac{x_{0} a_{l}}{\varepsilon}\left(e^{\varepsilon t}-e^{\varepsilon T}\right)\right) .
$$

Using the L'Hospital's rule, we get $\lim \sup _{t \rightarrow \infty} h(t) / t \leq \varepsilon / a_{l}$. Since $\varepsilon$ is arbitrary and $x(t, \omega)>0(t>0)$, we can obtain that $\lim _{t \rightarrow \infty} \int_{0}^{t} x(s, \omega) \mathrm{d} s / t=0$. 
Now, we will prove $\lim _{t \rightarrow \infty} \int_{0}^{t} u(s, \omega) \mathrm{d} s / t=0$. Dividing both sides of (13) by $t$, we get

$$
\begin{aligned}
\frac{1}{t} \int_{0}^{t} u(s, \omega) \mathrm{d} s & \leq \frac{f_{u}}{e_{l} t} \int_{0}^{t} x(s, \omega) \mathrm{d} s-\frac{u(t, \omega)}{e_{l} t}+\frac{u_{0}}{e_{l} t} \\
& \leq \frac{f_{u}}{e_{l} t} \int_{0}^{t} x(s, \omega) \mathrm{d} s+\frac{u_{0}}{e_{l} t}
\end{aligned}
$$

From $\lim _{t \rightarrow \infty} \int_{0}^{t} x(s, \omega) \mathrm{d} t / t=0$, letting $t \rightarrow \infty$ we obtain $\lim _{t \rightarrow \infty} \int_{0}^{t} u(s, \omega) \mathrm{d} t / t=0$. Since $\mathbb{P}\left(\Omega_{0}\right)=1$, this completes the proof of the theorem.

Theorem 4. If $e_{l}>0$ and $\widetilde{b}>0$, then species $x$ will be weakly persistent in the mean a.s.; that is, $\langle x\rangle^{*}>0$ a.s.

Proof. We claim that $\Omega_{0} \subset\left\{\langle x\rangle^{*}>0\right\}$. If the claim is not true, then $\left\{\langle x\rangle^{*}=0\right\} \cap \Omega_{0} \neq \varnothing$. By the proof of Theorem 3, if $e_{l}>0$, we have $\langle u(t, \omega)\rangle^{*}=0$ for any $\omega \in\left\{\langle x\rangle^{*}=0\right\} \cap \Omega_{0}$. It is easy to see that

$$
\begin{aligned}
& \langle a(\gamma(t)) x(t, \omega)\rangle^{*} \leq a_{u}\langle x(t, \omega)\rangle^{*}=0, \\
& \langle c(\gamma(t)) u(t, \omega)\rangle^{*} \leq c_{u}\langle u(t, \omega)\rangle^{*}=0 .
\end{aligned}
$$

From (12), we get

$$
\begin{aligned}
\frac{\ln x(t, \omega)}{t}= & \frac{\ln x_{0}}{t}+\frac{1}{t} \int_{0}^{t} b(\gamma(s)) \mathrm{d} s \\
& -\frac{1}{t} \int_{0}^{t} a(\gamma(s)) x(s, \omega) \mathrm{d} s \\
& -\frac{1}{t} \int_{0}^{t} c(\gamma(s)) u(s, \omega) \mathrm{d} s+\frac{M(t, \omega)}{t} .
\end{aligned}
$$

Combining this equation with (14) and (22), we have

$$
\limsup _{t \rightarrow \infty} \frac{\ln x(t, \omega)}{t}=\limsup _{t \rightarrow \infty} \frac{1}{t} \int_{0}^{t} b(\gamma(s)) \mathrm{d} s=\widetilde{b}
$$

Hence, there are positive constant $T_{0}$ and time sequence $\left\{t_{n}\right\}$ with $t_{n} \geq T_{0}$ and $t_{n+1}-t_{n} \geq 1$ for all $n \geq 1$ such that

$$
\begin{aligned}
\frac{\ln x\left(t_{n}, \omega\right)}{t_{n}}>\frac{\widetilde{b}}{2}, \\
\frac{|M(t, \omega)|}{t}<\frac{\widetilde{b}}{8},
\end{aligned}
$$

$$
\forall t>T_{0} .
$$

Let $\bar{b}=\max _{1 \leq i \leq n}\{|b(i)|\}$. Any positive constant $\Delta t<$ $\min \left\{1, t_{1} \widetilde{b} /(8 \bar{b})\right\}$ from (12) leads to

$$
\begin{aligned}
& \ln x\left(t_{n}, \omega\right)-\ln x(t, \omega) \\
& \quad \leq \int_{t}^{t_{n}} b(\gamma(s)) \mathrm{d} s+M\left(t_{n}, \omega\right)-M(t, \omega) \\
& \quad \leq \bar{b} \Delta t+\frac{\tilde{b}}{4} t_{n},
\end{aligned}
$$

for all $t \in\left[t_{n}-\Delta t, t_{n}\right]$. Combining with (25), we obtain

$$
\begin{aligned}
\ln x(t, \omega) & \geq \ln x\left(t_{n}, \omega\right)-\bar{b} \Delta t-\frac{\widetilde{b}}{4} t_{n} \geq \frac{\widetilde{b}}{4} t_{n}-\bar{b} \Delta t \\
& \geq \frac{\widetilde{b}}{8} t_{n}, \quad \forall t \in\left[t_{n}-\Delta t, t_{n}\right], n \geq 1 .
\end{aligned}
$$

Consequently,

$$
\begin{aligned}
\frac{1}{t_{n}} \int_{t_{1}}^{t_{n}} x(s, \omega) \mathrm{d} s & \geq \frac{1}{t_{n}} \sum_{t_{1}<t_{m} \leq t_{n}} \int_{t_{m}-\Delta t}^{t_{m}} x(s, \omega) \mathrm{d} s \\
& \geq \frac{1}{t_{n}} \sum_{t_{1}<t_{m} \leq t_{n}} \Delta t \exp \left\{\frac{\tilde{b}}{8} t_{m}\right\} \\
& \geq \frac{\Delta t}{t_{n}} \exp \left\{\frac{\tilde{b}}{8} t_{n}\right\} .
\end{aligned}
$$

Since $\tilde{b}>0$, $\lim \sup _{t \rightarrow \infty} \int_{t_{1}}^{t_{n}} x(s, \omega) \mathrm{d} s / t_{n}=+\infty$, which contradicts with $\omega \in\left\{\langle x\rangle^{*}=0\right\} \cap \Omega_{0}$. Therefore, $\Omega_{0} \subset$ $\left\{\langle x\rangle^{*}>0\right\}$; that is, $\langle x\rangle^{*}>0$ a.s.

Theorem 5. If $a_{l}>0, e_{l}>0, f_{l}>0$ and $\widetilde{b}>0$, then system (2) will be uniform permanent in the mean a.s. Moreover,

$$
\begin{aligned}
& \underline{x} \leq\langle x\rangle_{*} \leq\langle x\rangle^{*} \leq \bar{x}, \\
& \underline{u} \leq\langle u\rangle_{*} \leq\langle u\rangle^{*} \leq \bar{u}
\end{aligned}
$$

a.s.,

where $\underline{x}=\widetilde{b} e_{l} /\left(a_{u} e_{l}+c_{u} f_{u}\right), \underline{u}=f_{l} e_{l} \widetilde{b} / e_{u}\left(a_{u} e_{l}+c_{u} f_{u}\right)$, $\bar{x}=\tilde{b}\left(e_{u}\left(a_{u} e_{l}+c_{u} f_{u}\right)-c_{l} f_{l} e_{l}\right) / a_{l} e_{u}\left(a_{u} e_{l}+c_{u} f_{u}\right)$, and $\bar{u}=$ $f_{u} \tilde{b}\left(e_{u}\left(a_{u} e_{l}+c_{u} f_{u}\right)-c_{l} f_{l} e_{l}\right) / a_{l} e_{l} e_{u}\left(a_{u} e_{l}+c_{u} f_{u}\right)$.

Proof. From (13), we have

$$
\begin{aligned}
\int_{0}^{t} e(s) u(s) \mathrm{d} s & =\int_{0}^{t} f(s) x(s) \mathrm{d} s-u(t)+u_{0} \\
& \leq f_{u} \int_{0}^{t} x(s) \mathrm{d} s+u_{0} .
\end{aligned}
$$

Consequently, we have

$$
\int_{0}^{t} u(s) \mathrm{d} s \leq \frac{f_{u}}{e_{l}} \int_{0}^{t} x(s) \mathrm{d} s+\frac{u_{0}}{e_{l}} .
$$

For any $\varepsilon>0$ and $\omega \in \Omega_{0}$, there is $T$ such that

$$
\begin{gathered}
\frac{1}{t} \int_{0}^{t} b(\gamma(s)) \mathrm{d} s>\widetilde{b}-\frac{\varepsilon}{2}, \\
\frac{M(t)}{t}>-\frac{\varepsilon}{2},
\end{gathered}
$$


Substituting these inequalities and (31) into (12), we get

$$
\begin{array}{r}
\ln x(t)-\ln x_{0} \geq v t-\left(a_{u}+\frac{c_{u} f_{u}}{e_{l}}\right) \int_{0}^{t} x(s) \mathrm{d} s-\frac{c_{u} u_{0}}{e_{l}} \\
\forall t \geq T,
\end{array}
$$

where $v=\widetilde{b}-\varepsilon$. Let $g(t)=\int_{0}^{t} x(s) \mathrm{d} s$; then, we have

$$
\ln \frac{\mathrm{d} g(t)}{\mathrm{d} t}-\ln x_{0} \geq v t-\frac{c_{u} u_{0}}{e_{l}}-\left(a_{u}+\frac{c_{u} f_{u}}{e_{l}}\right) g(t) .
$$

In a similar discussion with Theorem 3 , we can obtain $\langle x\rangle_{*} \geq$ $\nu e_{l} /\left(a_{u} e_{l}+c_{u} f_{u}\right)$. Since $\varepsilon$ is arbitrary, we obtain $\langle x\rangle_{*} \geq$ $\widetilde{b} e_{l} /\left(a_{u} e_{l}+c_{u} f_{u}\right):=\underline{x}$ for all $\omega \in \Omega_{0}$.

Now, we will prove $\langle u\rangle_{*}$ also has a lower bound. From the above proof, we can imply for any $\varepsilon>0$ and $\omega \in \Omega_{0}$ that there is a positive constant $T$ such that $\int_{0}^{t} x(s) \mathrm{d} s \geq(\underline{x}-\varepsilon) t$ for all $t \geq T$. Substituting this inequality into (13), we have $u(t) \geq-e_{u} \int_{0}^{t} u(s) \mathrm{d} s+f_{l}(\underline{x}-\varepsilon) t$ for all $t \geq T$. Let $h(t)=$ $\int_{0}^{t} u(s) \mathrm{d} s$; then, we have $\mathrm{d} h(t) / \mathrm{d} t \geq-e_{u} h(t)+f_{l}(\underline{x}-\varepsilon) t$ for all $t \geq T$. Consider the following comparison equation $\mathrm{d} y(t) / \mathrm{d} t=-e_{u} y(t)+f_{l}(\underline{x}-\varepsilon) t$, with initial value $y(T)=h(T)$. By the well-known variation-of-constants formula, we have

$$
\begin{gathered}
y(t)=f_{l}(\underline{x}-\varepsilon) \int_{T}^{t} s \exp \left\{-e_{u}(t-s)\right\} \mathrm{d} s+h(T) \\
\cdot \exp \left\{-e_{u}(t-T)\right\}=\frac{f_{l}}{e_{u}}(\underline{x}-\varepsilon) \\
\cdot\left(\left(t-\frac{1}{e_{u}}\right)-\left(T-\frac{1}{e_{u}}\right) \exp \left\{-e_{u}(t-T)\right\}\right) \\
+h(T) \exp \left\{-e_{u}(t-T)\right\} .
\end{gathered}
$$

By the comparison theorem, we have that lim inf ${ }_{t \rightarrow \infty} h(t) / t \geq$ $\lim \inf _{t \rightarrow \infty} y(t) / t=f_{l}(\underline{x}-\varepsilon) / e_{u}$. Since $\varepsilon$ is arbitrary, we obtain that

$$
\langle u\rangle_{*}=\liminf _{t \rightarrow \infty} \frac{h(t)}{t} \geq \frac{f_{l}}{e_{u}} \underline{x}:=\underline{u} .
$$

In the following, we will prove the upper bound of $\langle x\rangle^{*}$ and $\langle u\rangle^{*}$. From (14) and (36), for any $\varepsilon>0$ and $\omega \in \Omega_{0}$, there exists a positive constant $T_{0}$ such that

$$
\begin{aligned}
\int_{0}^{t} b(s) \mathrm{d} s & \leq\left(\widetilde{b}+\frac{c_{l} \varepsilon}{3}\right) t \\
\int_{0}^{t} u(s) \mathrm{d} s & \geq\left(\underline{u}-\frac{\varepsilon}{3}\right) t \\
M(t) & \leq \frac{c_{l} \varepsilon}{3} t
\end{aligned}
$$

for all $t \geq T_{0}$. Substituting (37) into (12), we have

$$
\ln x(t)-\ln x_{0} \leq\left(\tilde{b}-c_{l}(\underline{u}-\varepsilon)\right) t-a_{l} \int_{0}^{t} x(s) \mathrm{d} s,
$$

Let $k(t)=\int_{0}^{t} x(s) \mathrm{d} s$; then, we have $\ln (\mathrm{d} k(t) / \mathrm{d} t)-\ln x_{0} \leq$ $\rho t-a_{l} k(t)$ for all $t \geq T_{0}$, where $\rho=\tilde{b}-c_{l}(\underline{u}-\varepsilon)$. In a similar discussion with Theorem 3 , we consequently have $\langle x\rangle^{*} \leq \rho / a_{l}$. Since $\varepsilon$ is arbitrary, we obtain

$$
\langle x\rangle^{*} \leq \frac{\tilde{b}-c_{l} \underline{u}}{a_{l}}:=\bar{x} .
$$

Rewriting (13), we have

$$
\begin{aligned}
\int_{0}^{t} u(s) \mathrm{d} s & \leq \frac{f_{u}}{e_{l}} \int_{0}^{t} x(s) \mathrm{d} s-\frac{u(t)}{e_{l}}+\frac{u_{0}}{e_{l}} \\
& \leq \frac{f_{u}}{e_{l}} \int_{0}^{t} x(s) \mathrm{d} s+\frac{u_{0}}{e_{l}} .
\end{aligned}
$$

Combine this inequality with (39), we have $\langle u\rangle^{*} \leq f_{u} \bar{x} / e_{l}:=$ $\bar{u}$. This completes the proof.

\section{Remarks}

In Theorem 4, we used a new method to study the weakly persistent in the mean of species $x$. If $c(i) \equiv 0$ for all $i \epsilon$ $\mathbb{S}$, system (2) becomes a stochastic logistic system without feedback control under the regime switching which has been studied by [10]. By Theorem 4, species $x$ is weakly persistent in the mean if $\widetilde{b}>0$, and the condition $a_{l}>0$ is not necessary. Hence, Theorem 3 in [10] is improved by Theorem 4 in this paper.

In [10], the authors studied system (2) without the feedback control and obtained the critical value between weak persistence and extinction; that is, species $x$ is weakly persistent if $\widetilde{b}>0$ and goes to extinction if $\widetilde{b}<0$. Hence, from Theorems $2-5$, we can find that if $e_{l}$ and $f_{l}$ are positive in system (2), species $x$ has the same extinction and permanence property as the system without feedback control under the same conditions. Therefore, the feedback control is harmless to the permanence of species even under the white and color noise perturbation environments.

\section{Numerical Simulation}

In this section, we will introduce some examples to illustrate our main results. Considering system (2), we can choose that the $\gamma(t)$ is a Markov chain with state space $\mathbb{S}=\{1,2\}$ and generator

$$
Q=\left(\begin{array}{cc}
-q_{12} & q_{12} \\
q_{21} & -q_{21}
\end{array}\right)
$$

$r(1)=0.4, r(2)=0.3, a(\gamma(t)) \equiv 0.01, c(\gamma(t)) \equiv 0.01, e(t) \equiv$ $0.1, f(t) \equiv 0.1, \sigma^{2}(1)=0.5$, and $\sigma^{2}(2)=1$; then, $b(1)=r(1)-$ $\sigma^{2}(1) / 2=0.15>0$ and $b(2)=r(2)-\sigma^{2}(2) / 2=-0.2<0$.

Example 1. We choose $q_{12}=1$ and $q_{21}=0$. Obviously, we have $\pi_{1}=0, \pi_{2}=1$, and $\widetilde{b}=b(2)=-0.2<0$. By Theorem 2 , we have that system (2) is almost surely extinct. 
Example 2. Let $q_{12}=0.4$ and $q_{21}=0.6$. Then,

$$
\begin{aligned}
& \pi_{1}=\frac{q_{21}}{q_{12}+q_{21}}=0.6, \\
& \pi_{2}=\frac{q_{12}}{q_{12}+q_{21}}=0.4,
\end{aligned}
$$

and $\tilde{b}=\pi_{1} b(1)+\pi_{2} b(2)=0.01>0$. By Theorem 5 , system (2) will be uniform permanent in the mean a.s.

From the two examples, we can find that the species is in the good environment when state $i=1$, and it is in the bad environment when state $i=2$. If the species stay more time in state $i=1$, it may be persistent even though $b(2)<0$. On the contrary, the species may go to extinction almost surely. Therefore, we can change the dynamic property of system (2) through changing the generator $Q$, but the feedback control is harmless to the permanence of species.

\section{Conflict of Interests}

The authors declare that there is no conflict of interests regarding the publication of this paper.

\section{Acknowledgment}

This work was supported by the National Natural Science Foundation of China (Grant no. 11401382), the Science Foundation for the Excellent Youth Scholars of Shanghai Municipal Education Commission (Grant no. 5113341105), and Hujiang Foundation of China (Grant no. B14005).

\section{References}

[1] M. A. Aizerman and F. R. Gantmacher, Absolute Stability of Regulator Systems, Holden Day, San Francisco, Calif, USA, 1964.

[2] F. D. Chen, "Global stability of a single species model with feedback control and distributed time delay," Applied Mathematics and Computation, vol. 178, no. 2, pp. 474-479, 2006.

[3] K. Gopalsamy and P. X. Weng, "Feedback regulation of logistic growth," International Journal of Mathematics and Mathematical Sciences, vol. 16, no. 1, pp. 177-192, 1993.

[4] H. Hu, Z. Teng, and S. Gao, "Extinction in nonautonomous Lotka-Volterra competitive system with pure-delays and feedback controls," Nonlinear Analysis: Real World Applications, vol. 10, no. 4, pp. 2508-2520, 2009.

[5] H. Hu, Z. Teng, and H. Jiang, "On the permanence in nonautonomous Lotka-Volterra competitive system with puredelays and feedback controls," Nonlinear Analysis. Real World Applications, vol. 10, no. 3, pp. 1803-1815, 2009.

[6] N. H. Du, R. Kon, K. Sato, and Y. Takeuchi, "Dynamical behavior of Lotka-Volterra competition systems: non-autonomous bistable case and the effect of telegraph noise," Journal of Computational and Applied Mathematics, vol. 170, no. 2, pp. 399-422, 2004.

[7] N. H. Du, R. Kon, K. Sato, and Y. Takeuchi, "Dynamical behavior of Lotka-Volterra competition systems: non-autonomous bistable case and the effect of telegraph noise," Journal of Computational and Applied Mathematics, vol. 170, no. 2, pp. 399-422, 2004.
[8] T. C. Gard, "Stability for multispecies population models in random environments," Nonlinear Analysis: Theory, Methods \& Applications, vol. 10, no. 12, pp. 1411-1419, 1986.

[9] L. Liu and Y. Shen, "New criteria on persistence in mean and extinction for stochastic competitive Lotka-Volterra systems with regime switching," Journal of Mathematical Analysis and Applications, vol. 430, no. 1, pp. 306-323, 2015.

[10] M. Liu, W. Li, and K. Wang, "Persistence and extinction of a stochastic delay logistic equation under regime switching," Applied Mathematics Letters, vol. 26, no. 1, pp. 140-144, 2013.

[11] Q. Luo and X. Mao, "Stochastic population dynamics under regime switching," Journal of Mathematical Analysis and Applications, vol. 334, no. 1, pp. 69-84, 2007.

[12] Q. Luo and X. Mao, "Stochastic population dynamics under regime switching II," Journal of Mathematical Analysis and Applications, vol. 355, no. 2, pp. 577-593, 2009.

[13] Y. Takeuchi, N. H. Du, N. T. Hieu, and K. Sato, "Evolution of predator-prey systems described by a Lotka-Volterra equation under random environment," Journal of Mathematical Analysis and Applications, vol. 323, no. 2, pp. 938-957, 2006.

[14] L. Zu, D. Jiang, and D. O’Regan, “Conditions for persistence and ergodicity of a stochastic Lotka-Volterra predator-prey model with regime switching," Communications in Nonlinear Science and Numerical Simulation, vol. 29, no. 1-3, pp. 1-11, 2015.

[15] X. Mao, Stochastic Differential Equations and Applications, Horwood Publishing Limited, Chichester, UK, 1997.

[16] N. Ikeda, Stochastic Differential Equations and Diffusion Processes, North Holland, Amsterdam, The Netherlands, 1981.

[17] X. Li, A. Gray, D. Jiang, and X. Mao, "Sufficient and necessary conditions of stochastic permanence and extinction for stochastic logistic populations under regime switching," Journal of Mathematical Analysis and Applications, vol. 376, no. 1, pp. 1128, 2011. 


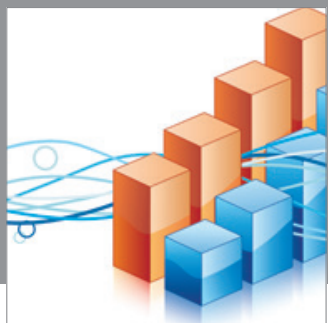

Advances in

Operations Research

mansans

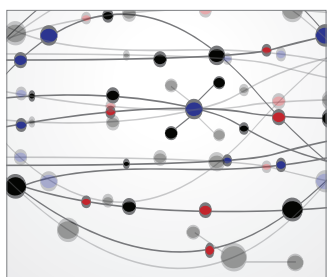

The Scientific World Journal
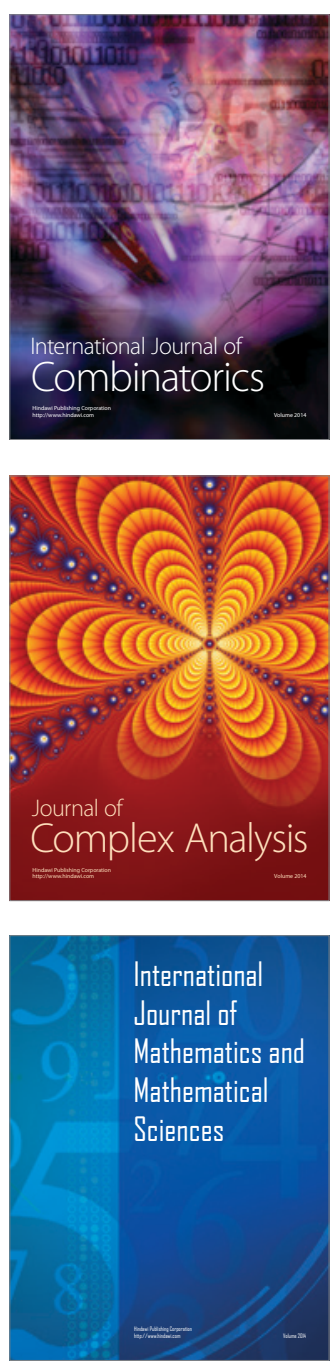
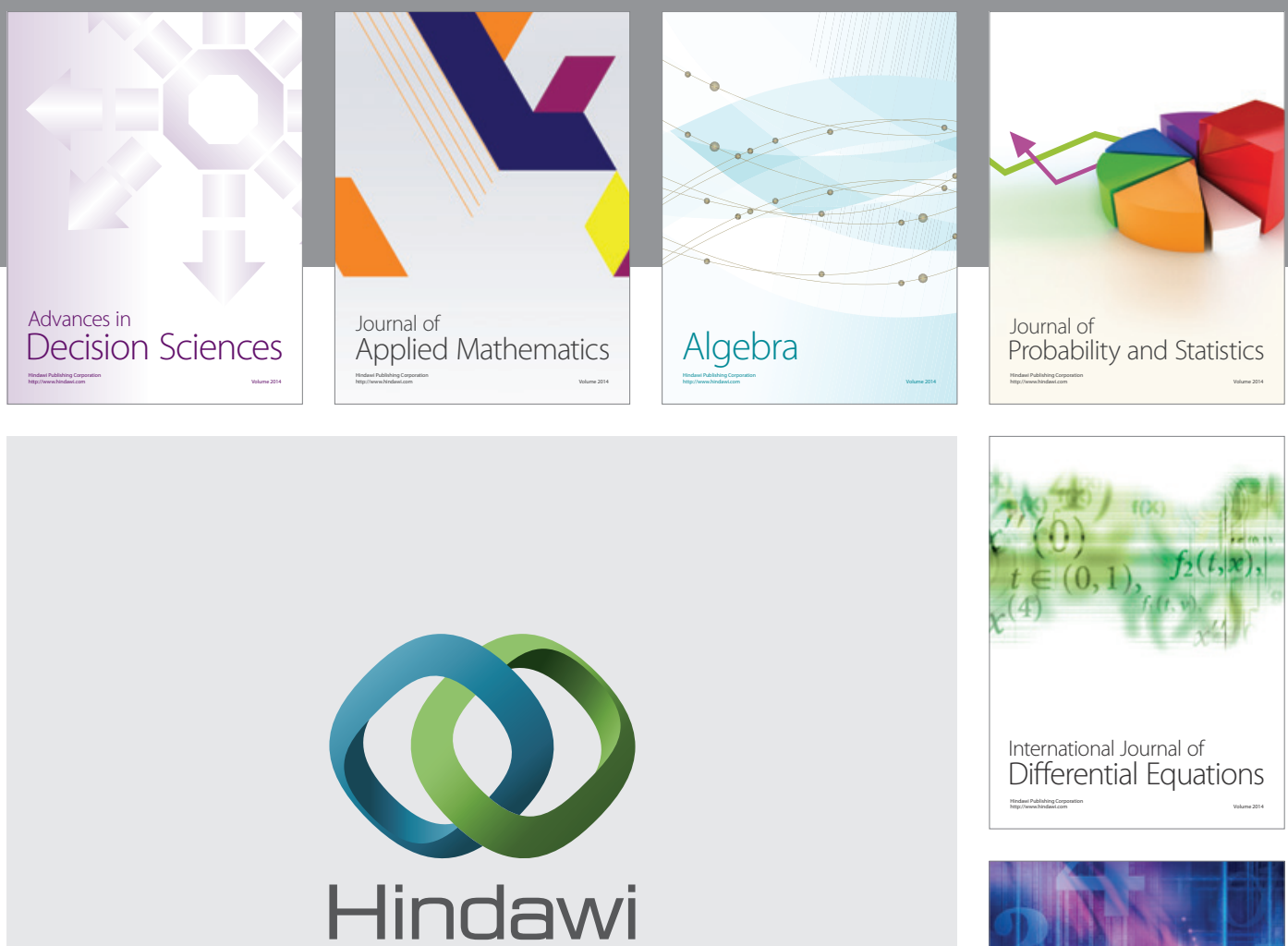

Submit your manuscripts at http://www.hindawi.com
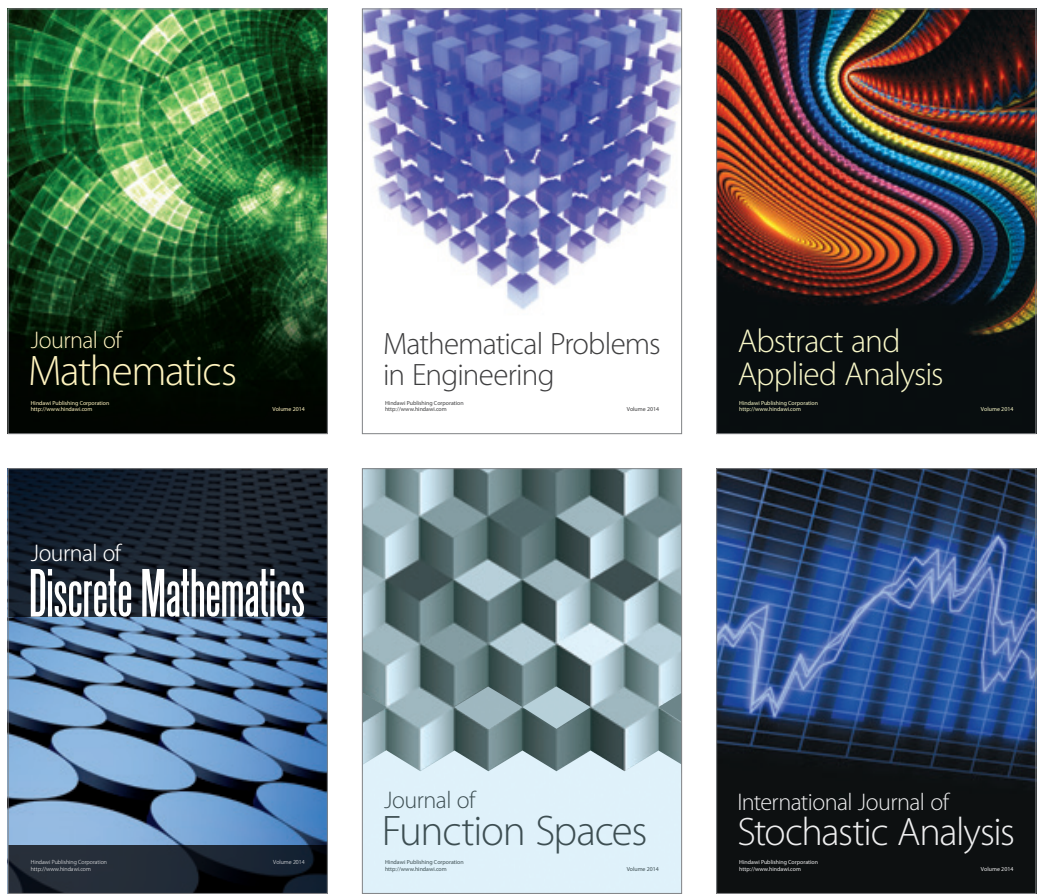

Journal of

Function Spaces

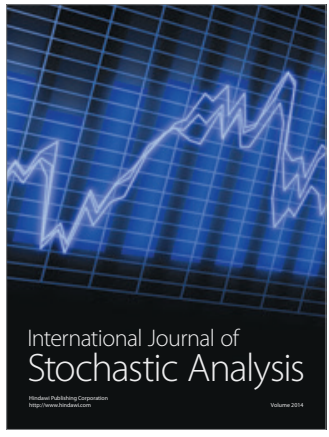

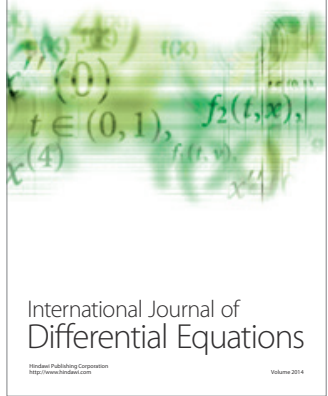
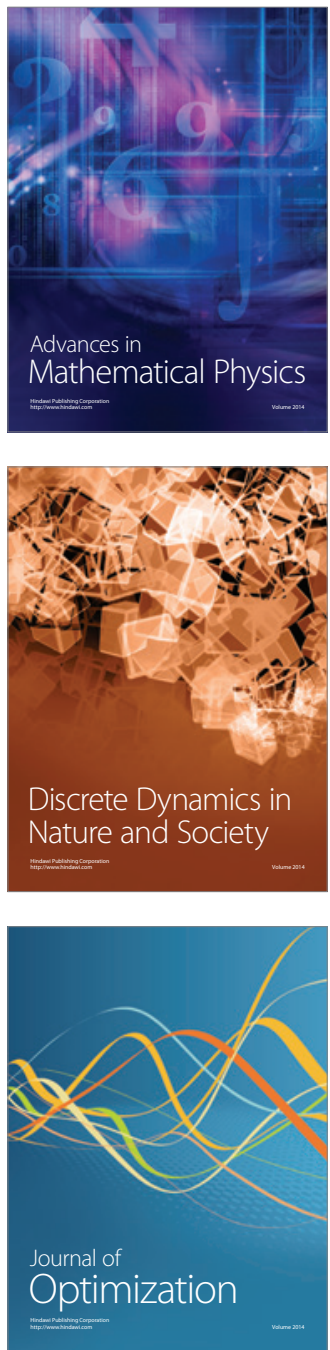